REVIEW

\title{
Is antibiotic resistance a problem? A practical guide for hospital clinicians
}

\author{
G Barlow, D Nathwani
}

Postgrad Med J 2005;81:680-692. doi: 10.1136/pgmi.2005.035113

Antibiotic resistance is an important concern for patients, physicians, healthcare managers, and policymakers. Inappropriate antimicrobial prescribing fuels the evolution of resistance, while poor basic hygiene facilitates the spread of resistant microbes between patients and healthcare staff. The development of infection with a resistant pathogen may lead to poorer health and economic outcomes. The problem for the frontline clinician, however, is how to balance the responsibility of prudent prescribing with the risk of sub-optimally treating a patient who may be infected with a resistant pathogen. This article discusses how hospital physicians can use severity and risk factor assessment, and knowledge of local microbial epidemiology, to guide empiric antibiotic prescribing. Most patients hospitalised with a community acquired bacterial infection in the UK can still be managed with a traditional first line antibiotic(s). In contrast, regimens that account for resistance are often required in patients with hospital acquired infections, particularly if the patient is critically ill.

See end of article for authors' affiliations

Correspondence to: Dr G Barlow, Department of Infection and Tropical Medicine, Castle Hill Hospital, Cottingham, East Yorkshire HU16 5JQ, UK; gavin.barlow@hey.nhs.uk

Submitted 15 March 2005 Accepted 20 April 2005
M icrobial resistance to antibiotics is not a new phenomenon. After the first use of penicillin in the 1950s, resistance developed rapidly in some bacteria (for example, Staphylococcus aureus) leading to clinical failure. ${ }^{1}$ This acquired resistance occurred because of Darwinian natural selection of resistant bacteria as a result of applying a new pressure to the microbial environment. In contrast, some bacteria are naturally (intrinsically) resistant to certain antibiotics (for example, penicillin resistance in Klebsiella pneumoniae). Widespread inappropriate antibiotic prescribing by healthcare professionals fuels the development of acquired resistance. In hospitals and long term care facilities, the spread of resistance is further encouraged by poor basic hygiene (for example, hand washing). ${ }^{2}$ In recent years, the consequences of this sub-optimal practice on patient and economic outcomes have become more apparent to patients, doctors, healthcare managers, and policymakers. This has culminated in the publication of a number of antibiotic resistance documents at national and international level, which urge prudent antibiotic prescribing. ${ }^{3}$ This crisis is heightened by the lack of new antimicrobial development, to combat emerging resistance, by the pharmaceutical industry.
The problem for the non-specialist hospital clinician at the "coal face", however, is how to balance the ecological and public health responsibility of antimicrobial prescribing with the risk of sub-optimally treating an individual patient who is acutely unwell and potentially infected with a pathogen resistant to the usual first line therapy. This balance can be difficult to achieve given the evidence showing critically ill patients have poorer health outcomes if they receive suboptimal antibiotic treatment. ${ }^{6-8}$ Unsurprisingly, when treating ill patients therefore, clinicians tend towards a "just in case" approach, which inevitably results in some patients receiving unnecessary intravenous and broad spectrum antimicrobial agents. Furthermore, the evolving culture of medicolegal litigation encourages clinicians to adopt a liberal and broad brush approach to antibiotic treatment and prophylaxis. This in turn can lead to poorer outcomes from complications of treatment and the induction of acquired resistance. ${ }^{9}$ This article will focus on the importance of antibiotic resistance in infections commonly managed in infectious disease and acute medical admissions units and by clinicians on-call for general medicine. Although the article is aimed at clinical practice in the UK, many of the principles described will be relevant to the management of patients in other countries. The primary aims of this review are to: (1) consider the impact of clinicians prescribing practice on antibiotic resistance and transmission; (2) examine the impact of resistance in specific infections on clinical and other outcomes; and (3) provide guidance regarding the key components of clinical decision making related to antibiotic resistance and prescribing empiric antibiotic treatment. To facilitate this process, where possible, we have used clinical case scenarios based on our experience and the available literature.

\section{METHODS}

Infections commonly admitted from the community and other hospital wards were identified from a previous audit of admissions to Tayside's regional infection unit. ${ }^{10}$ Most patients admitted to this teaching hospital based unit are either

Abbreviations: MRSA, methicillin resistant Straphyloccus aureus; MSSA, methicillin sensitive Straphyloccus aureus; PRP, penicillin resistant pneumococci; MIC, minimum inhibitory concentration; SIRS, systemic inflammatory response syndrome; $C A P$, community acquired pneumonia; HAP, hospital acquired pneumonia; BTS, British Thoracic Society; COPD, chronic obstructive pulmonary disease; TB, tuberculosis; UTI, urinary tract infection; ESBL, extended spectrum $\beta$-lactamases 
transferred from the hospital's acute medical admissions unit or directly admitted from the community via general practitioners. Other patients are admitted via accident and emergency or from other hospital departments. A literature search using Pubmed and the Cochrane Controlled Trials Database was performed using the keywords: "resistance" and "resistant" combined with the infection (for example, gastroenteritis) or pathogen (for example, Staphylococcus aureus). Other articles and book chapters were identified from the authors' collections. Data about the rates of resistance in individual pathogens were ascertained from the UK's Health Protection Agency's web site. ${ }^{11}$

\section{DOES MY PRACTICE INFLUENCE THE DEVELOPMENT AND SPREAD OF RESISTANCE?}

Consider the following story: Mrs White, a 70 year old previously well woman, is admitted to your hospital's acute medical admissions unit. On examination her MSQ is 6/10 (normally 10/10), but she is otherwise well, able to take oral medication, and without any positive systemic inflammatory response syndrome (SIRS) markers. Urine dipstick testing shows $1+$ of blood and $1+$ of protein, but no leucocytes or nitrites. On the post-take ward round you cannot find an obvious cause for her confusion, but the nurse mentions that she has been incontinent of urine on a number of occasions and that her urine is "smelly". You therefore feel a urinary tract infection is the most probable explanation of her symptoms. You prescribe oral cefalexin $500 \mathrm{mg} / 6 \mathrm{~h}$ in keeping with the hospital's antibiotic policy.

After taking the first dose, although most of the drug is absorbed into her bloodstream, some remains in the gastrointestinal tract exposing a previously healthy and diverse population of commensal bacteria to varying concentrations of antibiotic. ${ }^{2}$ Most of the commensals die, but two in particular, Clostridium difficile and a vancomycin resistant enterococcus, are resistant to cefalexin and thrive in this new and competitor-free environment. ${ }^{12}$ A small number of other bacteria are able to adapt or genetically mutate to protect themselves. On the patient's skin, many of the patient's commensal bacteria have also been killed and replaced by more resistant bacteria from the hospital's environment.

Mrs White has been housed in an open bay bed next to another woman who has been admitted to hospital from a residential home with leg ulcers. Although this patient has been screened for methicillin resistant Staphyloccus aureus (MRSA), the results are awaited and healthcare staff are unaware that she is colonised with MRSA. The nurse looking after Mrs White is having a particularly busy day and forgets to wash her hands in between touching the two patients during your ward round; Mrs White's skin is rapidly colonised with MRSA.

Mrs White's confusion gradually resolves. It transpires that she had forgotten to get a repeat prescription for diazepam, which she has been taking for her "nerves" for some years. Urine culture is negative and you stop the cefalexin. On the day before discharge, she develops profuse watery diarrhoea. She is moved to a side room, but she is incontinent of faeces before being transferred. As a result, the patient with the leg ulcers is now colonised with the vancomycin resistant enterococcus. You suspect cefalexin induced Clostridium difficile related diarrhoea and start empiric oral metronidazole. The diagnosis is confirmed by detection of toxin in a stool sample.

After a few days of intravenous fluid treatment, the skin around the peripheral intravenous catheter becomes tender and erythematous. The catheter is removed and the tip sent for culture; MRSA is isolated. On occasions, she continues to have fever of $>38^{\circ} \mathrm{C}$ over the next two weeks. She begins to complain of back pain at the T10 level. A plain radiograph is normal, but vertebral osteomyelitis is confirmed by a MRI scan. MRSA is cultured from a computed tomography (CT) guided biopsy specimen. She is prescribed a six week course of antibiotics, initially intravenous vancomycin and then oral rifampicin and trimethroprim. The clostridium diarrhoea recurs with the second regimen, which further delays her recovery and discharge. Eventually she is able to return to her residential home. What she and others do not realise, however, is that she is now harbouring many bacteria that have either taken the first steps on the resistance ladder or are resistant to first line antimicrobials. Potentially these can be transmitted to other residents and healthcare staff.

Although the above case may seem extreme, in the authors' experience, the complications described are not that unusual in hospital practice and were as a direct result of an initial well meaning, but inappropriate antibiotic prescription. A more prudent approach would have been to wait for the results of the urine culture before prescribing an antibiotic.

All antimicrobial prescribing has an impact on the individual patient and the local ecology, although the prescriber may not subsequently witness these consequences. For example, it has been shown that patients who have a trimethroprim resistant bacterium isolated from urine are more likely to have been exposed to trimethroprim or other antibiotics beforehand. ${ }^{13}$ Likewise, patients who have an amoxicillin resistant Haemophilus influenzae isolated from sputum are more likely to have been exposed to $\beta$-lactams or other antibiotics in the previous three months. ${ }^{14}$ These findings are supported by a number of epidemiological studies and observations showing associations between antibiotic prescribing and resistance. For example, a study from Wales showed an association between rates of GP antibiotic prescribing and trimethroprim resistance in urinary tract infections. ${ }^{15}$ In Finland, an increase in erythromycin prescribing was associated with an increase in erythromycin resistance in group A streptococci isolates. When erythromycin prescribing subsequently fell, so did resistance. ${ }^{16}$ An increase in vancomycin prescribing in hospitals in the USA seemed to lead to an increase in the incidence of vancomycin resistant enterococcal (VRE) infections. ${ }^{17}$ In European countries there is an inverse relation between rates of narrow spectrum antibiotic use and resistance. ${ }^{18}$ Although these associations do not prove a causal link, the available evidence is compelling.

\section{DOES ANTIBIOTIC RESISTANCE RESULT IN POORER OUTCOMES FOR PATIENTS?}

The impact of antibiotic resistance on health and economic outcomes has not been fully elucidated, primarily because of methodologically flawed studies. The expert consensus, based on the available published literature, however, is that infection with resistant pathogens does lead to poorer clinical and economic outcomes. ${ }^{19} 20$ Some of the evidence for two common pathogens is briefly reviewed below.

For MRSA there is clear evidence that infection negatively affects patient morbidity and mortality. For example, a recent meta-analysis of studies between 1980 and 2000 showed that MRSA bacteraemia results in higher mortality than methicillin sensitive Staphylococcus aureus (MSSA) (pooled odds ratio of six studies $=2.2$, 95\% CI 1.2 to $3.8 ; \mathrm{p}=0.007){ }^{21}$ There was minimal heterogeneity in this analysis and no evidence of publication bias. A number of other studies have shown the potential economic impact of MRSA infections. ${ }^{22}{ }^{23}$

In contrast, penicillin resistant pneumococci (PRP) do not seem to have an impact on outcome in patients hospitalised with pneumococcal pneumonia and/or bacteraemia; other factors such as severity at presentation are more 
important. ${ }^{24-26}$ This in part may be attributable to the low prevalence of high level penicillin resistance (minimum inhibitory concentration (MIC) $\geqslant 4 \mathrm{mg} / \mathrm{l}$ ), even in countries with overall high rates of pneumococcal resistance, such as Spain. ${ }^{26}$ Respiratory infections and bacteraemia caused by pneumococci of low or intermediate level resistance will still respond to high doses of penicillin because $\beta$-lactam agents (pencillins and cephalosporins) concentrate well in lung tissue, giving rise to concentrations well above the MIC of the bactena. In the community, however, the situation is less clear and probably depends on the background level of resistance to first line treatment (amoxicillin or erythromycin/clarithromycin in the UK) and the initial choice of agent. Singer et al recently developed a probability model to estimate the impact of resistance on outpatient treatment of pneumococcal pneumonia. ${ }^{27}$ They concluded that in countries with substantially reduced levels of susceptibility, antibiotic resistance contributes significantly to the subsequent need for hospitalisation (for example, greater than $40 \%$ of hospitalised cases in France). In the UK, however, where there is a low prevalence of penicillin and erythromycin pneumococcal resistance, resistance is less important. This emphasises the importance of adjusting first line treatment to local antibiotic resistance patterns.

\section{IDENTIFYING PATIENTS AT RISK OF INFECTION WITH A RESISTANT PATHOGEN}

In patients admitted from the community, to identify factors that are known to be associated with a higher risk of colonisation and infection with a resistant pathogen, a thorough history and scrutiny of the patient's case notes is required. For nosocomial infections, these factors are similar across a range of bacteria (see box 1) and seem to relate to the patient's direct or indirect exposure to hospitals. ${ }^{28}$ Hospital experienced patients and nursing home residents are usually readily identified from the GP's letter, the history, or the case notes. For pathogens that are usually community acquired (for example, the pneumococcus), knowledge of specific risk factors (for example, travel to Spain or another endemic region) is also important. Increasingly there are reports of infections that are typically hospital acquired (for example, MRSA) being acquired in the community by patients without recognised risk factors. ${ }^{29}{ }^{30}$ In the UK, this is a rare but emerging problem.

In some patients, however, the identification of a risk factor is easily missed. For example, we have previously been involved in the care of a young male patient with severe community acquired pneumonia and empyema. He was

Box 1 Common risk factors for nosocomial colonisation/infection with resistant Staphylococcus aureus, enterococcus, Gram negative bacilli, Clostridium difficile, and Candida spp (adapted from Safdar and Maki ${ }^{28}$ )

- Advanced age

- Comorbidity

- Severity of illness

- Inter-institutional transfer of patients (especially from nursing homes)

- Prolonged hospitalisation

- Gastrointestinal or transplant surgery

- The presence of any invasive device (especially central venous catheters)

- Exposure to antibiotics (especially cephalosporins) initially managed with British Thoracic Society (BTS) recommended antibiotics, a chest drain, and intra-pleural streptokinase. Gram positive cocci were seen on a Gram stain from a sample of empyema fluid taken shortly after admission and flucloxacillin was added to the empiric antibiotic regimen. Perplexingly, MRSA was confirmed two days later. After taking the history again, the probable source of infection became clear; he worked in a nursing home. The failure to take an occupational history on the acute medical admissions unit and link this to the Gram stain findings and his severity of illness resulted in him not receiving antiMRSA antibiotics for over 48 hours after admission. He subsequently developed liver and kidney abscesses from which MRSA was also isolated.

Given the evidence linking antibiotic use at the population and individual level with an increased risk of subsequent infection with a resistant pathogen, ${ }^{13}{ }^{14}$ it is worth asking the patient about recent and past antibiotic exposure and reviewing the case notes to see what antibiotics previous infections have responded to (for example, in cystic fibrosis patients colonised with Pseudomonas spp). In patients recently exposed to the usual first line agent, it may be worth considering an alternative first line agent of another antibiotic class, particularly if the patient is seriously ill. Likewise, reviewing the patient's case notes and the hospital's results database may identify previous positive microbiological tests, which may influence antibiotic choice. Huang and Platt showed that 29\% of patients newly identified as being colonised or infected with MRSA, subsequently developed a MRSA infection over the next 18 months. ${ }^{31}$ The risk was higher $(40 \%-50 \%)$ for isolates from the nares or bones/joints compared with $20 \%-30 \%$ for the respiratory tract and soft tissue isolates. It should be remembered, however, that colonisation is only the first step in infection and that the previous isolation of, for example, Pseudomonas aeruginosa from a skin swab does not necessarily mean that it is the causal pathogen of a patient's cellulitis. The management of patients who are known to be colonised with a resistant pathogen is discussed further below.

A similar approach to clinical decision making has recently been used by a consensus group for prescribing empiric antibiotic treatment for suspected Gram positive infections in surgical patients. ${ }^{32}$ Core to this guidance is when to use glycopeptides (vancomycin or teicoplanin) for possible MRSA infection. The current lack of high quality evidence precludes a systematic review in this area. The decision tree guides clinicians in their choice of empiric antibiotic therapy by identifying key patient, treatment, and environmental risks for MRSA infection (see box 2).

\section{USING ADVERSE PROGNOSTIC INDICATORS AND CLINICAL JUDGEMENT IN GUIDING EMPIRIC ANTIBIOTIC TREATMENT}

When a physician sees a patient in clinical practice, the decision regarding the level of medical intervention required (for example, intravenous compared with oral and broad spectrum compared with narrow spectrum antibiotics) is usually based on clinical judgement. Validated prognostic tools, such as CURB65 for community acquired pneumonia, ${ }^{33}$ are increasingly available to support the clinical decision making process. CURB65, for example, can provide support for a physician's judgement that a patient with community acquired pneumonia can be managed as an outpatient with an oral antibiotic. Many of these tools, however, are either disease specific or require detailed data and are therefore difficult to use in clinical practice. A generic tool that requires minimal data and that can be applied at the bedside, in combination with clinical judgement, is the systemic inflammatory response syndrome (SIRS) (see box 3), ${ }^{34}$ 
Box 2 Core risk factors for MRSA colonisation/ infection in postoperative surgical patients (adpated from Solomkin et a ${ }^{32}$ )

\section{Patient related}

- Known colonisation

- Open, chronic skin wound or breakage

- Obesity

- End stage renal disease, diabetes mellitus, liver failure, or spinal injury

\section{Treatment related}

- Previous antibiotic therapy

- Hospital stay $>2$ weeks

- Invasive procedure or intravenous catheterisation, especially central venous lines

- Prolonged mechanical ventilation

\section{Environment}

- Long term care facility

- Prolonged hospitalisation, especially in high risk areas such as high dependency

Sepsis is the presence of SIRS because of infection. Patients with sepsis and clinical evidence of organ hypoperfusion are said to have severe sepsis. A patient with severe sepsis and hypotension (systolic BP $<90 \mathrm{~mm} \mathrm{Hg}$ ) that fails to respond to appropriate intravenous fluid therapy has septic shock. Mortality increases from sepsis through severe sepsis to septic shock. In combination with clinical judgement, these criteria can also be used to aid the decision about whether a patient is prescribed the usual first line antibiotic for any given infection (that is, a patient with risk factors for resistance, but no severity markers) or "upgraded" to an antibiotic(s) that will cover a suspected resistant pathogen (that is, a patient with a significant risk of a resistant pathogen and severe sepsis); box 4 shows a clinical example of this.

\section{WHAT TO DO ABOUT PATIENTS WHO HAVE "FAILED" ANTIBIOTIC TREATMENT}

There are many reasons why patients apparently fail antibiotic treatment; in the UK, resistance is a comparatively unusual cause. Perhaps the most obvious is that infection does not exist or is attributable to a pathogen unaffected by antibacterial agents (for example, a virus, fungi, protozoa, or helminth). A good example of this is the apparent failure of antibiotics in a patient with acute bronchitis because of a

Box 3 Definition of the systemic inflammatory response syndrome (SIRS) (adapted from Nyströ $m^{34}$ )

SIRS is present if two or more of the following exist:

- Temperature $<36^{\circ}$ or $>38^{\circ} \mathrm{C}$

- Heart rate $>90 /$ minute

- Respiratory rate $>20 / \mathrm{min}$

- White cell count $<4000$ cells $/ \mathrm{mm}^{3}$ or $>12000$ cells/ $\mathrm{mm}^{3}$

Sepsis is clinical evidence of infection (from the history or examination) plus SIRS as defined above
Box 4

A 67 year old woman is admitted to hospital with left lower limb cellulitis associated with a varicose ulcer. She has not responded to five days of oral flucloxacillin $250 \mathrm{mg}$ four times daily. She was found to be colonised with MRSA during a hospital admission for acute cholecystitis one year previously; she did not receive eradication treatment. She does not have any systemic symptoms (for example, rigors) and none of the SIRS criteria are positive.

1. What are the probable reasons for non-response to oral antibiotic treatment in this patient?

2. What is the most appropriate initial antibiotic regimen from the list below?

(All regimens are intravenous)

(A) Vancomycin $1 \mathrm{~g} / 12 \mathrm{~h}$

(B) Linezolid $600 \mathrm{mg} / 12 \mathrm{~h}$

(C) Flucloxacillin $1 \mathrm{~g} / 6 \mathrm{~h}$

(D) Clindamycin $600 \mathrm{mg} / 6 \mathrm{~h}$

(E) Ceftriaxone $1 \mathrm{~g} / 24 \mathrm{~h}$

Answer:

1. There are a number of reasons why this patient may not have responded to initial outpatient therapy. Firstly, she may not have been taking or adhering to her prescription. Secondly, a high enough antibiotic concentration may not be being achieved at the site of infection; it is worth noting the low dose of flucloxacillin. Thirdly, she may have chronic osteomyelitis. Finally, the infection may be attributable to MRSA or another flucloxacillin insensitive pathogen, although the other reasons should be excluded first. (See text for more detail).

2. Although the cellulitis may be attributable to MRSA, it could also be attributable to a methicillin sensitive Staphylococcus aureus (MSSA) or Streptococcus pyogenes. Given that the patient is not severely unwell, in the authors' opinion, the most appropriate initial antibiotic regimen is flucloxacillin (the narrowest spectrum and best anti-staphylococcal agent in the list above) in an adequate dose. This approach reduces the patient's (and the hospital's) exposure to vancomycin thereby reducing the risk of VRE emergence and, in the authors' experience, is often curative. If the patient does not respond to flucloxacillin or if she deteriorates then it is reasonable to consider an anti-MRSA agent at that stage.

respiratory virus (for example, influenza A). Another common cause of apparent failure is poor patient adherence because of adverse effects (for example, nausea) or other reasons (for example, chaotic lifestyle); although this is less probable if treatment is supervised as in the hospital setting. Even if the patient is taking the antibiotic regularly, the choice of antibiotic may be inappropriate for the probable causal bacteria (for example, ciprofloxacin for streptococcal cellulitis). Assuming a suitable agent has been chosen, the antibiotic concentration at the site of infection may be suboptimal. This may be because of under-dosing or poor tissue penetration. When treating patients with oral antibiotics, it is also important to consider the bioavailability of the drug (that is, how much of the orally taken dose gets into the bloodstream). For many oral $\beta$-lactam agents this is about 
$50 \%-70 \%$ of the same intravenous dose. ${ }^{35}$ If a patient is treated for cellulitis with oral flucloxacillin $500 \mathrm{mg}$ every six hours, only a proportion of each $500 \mathrm{mg}$ dose will get into the bloodstream and a smaller proportion will then get to the target site.

For $\beta$-lactam antibiotics (for example, penicillin, cephalosporins and penicillin/ $\beta$-lactamse inhibitor combinations, for example, co-amoxiclav), it is the time that the blood/tissue concentration of the antibiotic remains above the MIC of the bacterium that is associated with antibacterial effect (time dependent efficacy). ${ }^{36}$ This means that the dosing interval is more important than the dose and that increasing the dose of the agent will not increase antibacterial effect as long as it is high enough to reach a concentration above the MIC of the infecting pathogen. The half life of the drug will dictate how frequently it needs to be prescribed to maintain the concentration above the MIC. For fully sensitive pathogens (that is, pathogens with low MICs), British National Formulary recommended regimens should suffice (for example, amoxicillin $500 \mathrm{mg}$ every eight hours for fully sensitive pneumococci). For pathogens with higher MICs (for example, low and intermediate level penicillin resistant pneumococci), higher doses should be prescribed frequently (for example, amoxicillin $1 \mathrm{~g}$ every six hours). In clinical practice it is difficult to predict resistance at the point of admission to hospital and the MIC of any isolated pathogen is not usually measured. It is therefore better to routinely use high doses (for example, as recommended in the BTS's community acquired pneumonia guidelines ${ }^{37}$ ). Failure to prescribe adequate doses could induce antibiotic resistance as has been shown in pneumococci. ${ }^{38}$ In life threatening infection, it has been suggested that a lower dose $\beta$-lactam infusion would be better at maintaining the time above the MIC compared with intermittent higher doses ${ }^{39}$; potentially, this approach could also ameliorate the deterioration seen in some patients after the first dose of antibiotics because of pro-inflammatory mediator release. When using teicoplanin (a time dependent antibiotic with a long half life), to rapidly achieve and maintain a steady serum/tissue concentration, it is necessary to prescribe a loading dose; this is particularly important when treating patients with life threatening infections.

In contrast, with quinolone and aminoglycoside antibiotics it is the ratio between the maximum drug concentration $\left(\mathrm{C}_{\max }\right)$ and the MIC and the ratio between the area under the 24 hour antibiotic concentration time curve and the MIC $\left(\mathrm{AUC}_{24} / \mathrm{MIC}\right)$ that are related to antibacterial effect (concentration dependent efficacy). ${ }^{36}$ In this situation it is the dose of the antibiotic that is more important than the dosing interval in determining efficacy and the likelihood of emerging resistance (for example, once daily gentamicin dosing).

Even when the dose and dosing interval is appropriate and the pathogen's MIC low, some antibiotics penetrate certain tissues poorly. This may be because of antibiotic or patient factors. For example, vancomycin and teicoplanin are both large molecules and are known to penetrate certain tissues, in particular the lung, poorly. ${ }^{40}{ }^{41}$ In contrast, certain agents (for example, rifampicin and clindamycin in orthopaedic infections) are used because they have high bioavailability and penetrate tissues well. ${ }^{42}$ Patient factors include, for example, peripheral vascular disease or chronic soft tissue damage, which both reduce the amount of antibiotic getting to the site of infection. Other reasons for treatment failure are the presence of: polymicrobial infection that is not adequately covered by the antibiotic regimen; an abscess requiring surgical or radiological drainage; or a persistent focus of infection (for example, dead bone tissue in chronic osteomyelitis) requiring debridement.

\section{THE CLINICAL RELEVANCE OF ANTIBIOTIC RESISTANCE IN INFECTIONS COMMONLY ADMITTED TO THE ACUTE MEDICAL ADMISSIONS UNIT}

\section{Gastroenteritis}

Campylobacter and Salmonella spp are the two commonest causes of bacterial gastroenteritis in the UK. In 2000, resistance to ciprofloxacin in $C$ jejuni $(\mathrm{n}=5914)$ and $C$ coli $(\mathrm{n}=599)$ was $18 \%$ and $26 \%$, respectively. In contrast, resistance to erythromycin was $1 \%$ and $5 \%$, respectively. ${ }^{11}$ Campylobacter gastroenteritis is usually a self limiting illness and the vast majority of cases, even those requiring admission to hospital, do not require antibiotic therapy. When an antibiotic is prescribed (for example, for prolonged or severe illness), erythromycin or clarithromycin (not ciprofloxacin) are the agents of choice.

As with campylobacter, most salmonella infections are self limiting and do not require antibiotic therapy. Salmonella spp, however, have a greater predilection to causing bacteraemia and invasive infection (for example, abscess formation) than Campylobacter spp. Antibiotic therapy should therefore be considered in patients who have severe sepsis or who are at higher risk of poor outcome (the elderly ( $>60$ years old) and patients with: extensive atheromatous disease (for example, known aortic aneurysm) or a prosthetic heart valve, hypochlorhydria, inflammatory bowel disease, diabetes mellitus, renal impairment, rheumatological disease, and significant drug or disease induced immunosuppression) as recommended in UK guidelines. ${ }^{43}$ Resistance to ciprofloxacin in the two commonest Salmonella spp (enteritidis and typhimurium) was $11 \%$ and $12 \%$, respectively, in the year 2000 . Ciprofloxacin therefore remains the agent of choice. It should be noted, however, that certain Salmonella spp (for example, virchow and hadar) have higher rates of ciprofloxacin resistance ( $52 \%$ and $48 \%$, respectively). ${ }^{11}$

At a global level, multidrug resistance in gastroenteritis causing bacteria is of increasing concern. Imported resistance in Salmonella spp, for example, has been recognised for many years and ciprofloxacin resistance in $S$ typhi (the cause of typhoid fever) is increasing in endemic countries. ${ }^{44} 45$ Although it is important that clinicians in the UK are aware of this, it should rarely affect prescribing practice. If an invasive, resistant Salmonella spp (or other gastroenteritis causing bacteria) is suspected or confirmed, expert advice regarding management should be sought (from a microbiologist or infectious disease physician).

\section{Respiratory tract infections}

\section{Community acquired pneumonia}

There has been considerable debate about the impact of PRP on outcomes for patients with community acquired pneumonia (CAP). Guidance regarding empiric antibiotic therapy in national and specialist society guidelines in the USA, where there is a high incidence of CAP attributable to PRP, has subsequently been adjusted to include the new fluoroquinolone antibiotics (for example, levofloxacin or moxifloxacin) as first line agents for hospitalised patients. ${ }^{46-48}$ The published evidence, however, suggests that adequate doses of a $\beta$-lactam agent (for example, intravenous or oral amoxicillin $\mathrm{lg}$ three times daily) remain effective in the vast majority of cases of PRP CAP requiring hospital management. ${ }^{24-26}$

In the UK, the incidence of PRP has gradually increased from $<1 \%$ in 1990 to $7 \%$ in 2000 , although there is notable geographical variation. ${ }^{11}$ For example in London and north west England rates are higher at 13\% and 12\%, respectively. The BTS guidelines do not suggest changing prescribing practice in patients who have recently returned from a high incidence area (North America, the Southern Mediterranean, 
Eastern Europe, and developing countries). We believe that clinicians should only consider adding a new fluoroquinolone to one of the BTS recommended regimens in the few patients who have returned from a high incidence country, are severely ill and require high dependency or intensive care. The rationale for this is to cover the theoretical possibility of high level pneumococcal resistance affecting outcome in a cohort of patients who have most to lose by receiving suboptimal treatment. A desirable "side effect" of this approach is that patients also receive increased legionella cover, which has a higher incidence in severely ill patients with pneumonia returning from abroad.

Severe CAP can be recognised using a combination of clinical judgement and the CURB65 prognostic tool. ${ }^{33}$ This defines severe CAP (mortality $=20 \%$ ) as the presence on admission to hospital of three or more of: new confusion, urea $>7 \mathrm{mmol} / \mathrm{l}$, respiratory rate $\leqslant 30 /$ minute, systolic BP $<90 \mathrm{~mm} \mathrm{Hg}$ or diastolic $\mathrm{BP} \leqslant 60 \mathrm{~mm} \mathrm{Hg}$, and age over 65 years. Patients with 0 or 1 (mortality $<1 \%$ ) or 2 (mortality $=7 \%$ ) CURB65 criteria are defined as non-severe. Given the much lower mortality in this cohort and the available evidence, patients with non-severe CAP and risk factors for PRP should be treated initially with the usual BTS recommended antibiotics. ${ }^{37}$

\section{Exacerbations of chronic obstructive pulmonary disease (COPD)}

Streptococcus pneumoniae, Haemophilus influenzae, and Moraxella catarrhalis are the main bacteria associated with acute exacerbation of COPD. Although the incidence of PRP is low in the UK, penicillin resistance is considerably higher in Haemophilus and Moraxella spp. There is controversy, however, about what impact, if any, antibiotic treatment has on outcomes for patients with acute exacerbation of COPD, regardless of antibiotic resistance. The evidence suggests that those with increased sputum production, sputum purulence, and shortness of breath gain some benefit from receiving antibiotics. ${ }^{49}$ It would seem sensible therefore to target BTS recommended treatment $\left(\operatorname{amoxicillin}^{50}\right)$ at patients fulfilling these criteria, while reserving second line agents (for example, co-amoxiclav and old/new fluoroquinolones), to which organisms are less likely to be resistant, for the severely ill subset of patients (those requiring non-invasive or mechanical ventilation) and those failing to improve after a reasonable course (five days) of first line therapy.

\section{Tuberculosis}

Although resistance to antituberculous drugs is an important global issue, it is beyond the scope of this article to discuss this in detail. Multidrug resistant TB (Mycobacterium tuberculosis resistant to rifampicin and isoniazid) remains rare in the UK ( $1.1 \%$ in the UK in 2000). ${ }^{11}$ Resistance to either rifampicin $(1.5 \%)$ or isoniazid $(6.4 \%)$ alone is more common. It is important that clinicians initially managing suspected TB cases are aware of the main risk factors for MDR-TB: a patient from a developing country or Eastern Europe or a history of prior TB treatment, or both, especially if poorly adherent. If MDR-TB is suspected, the hospital's infection control team should be immediately contacted. One of the important principles of TB management is never to add a single antituberculous drug to a failing regimen. It is therefore important that a physician experienced in the management of MDR-TB (a respiratory or infectious disease physician) is involved in the care of such patients from a very early stage. Further information about the antimicrobial management of MDRTB is available from a recent review article. $^{51}$

\section{Skin and soft tissue infections}

Streptococcus pyogenes (Lancefield group A streptococcus) and MSSA cause most community acquired skin and soft tissue infections in the UK. Streptococcus pyogenes is always sensitive to penicillin. High dose flucloxacillin monotherapy (1-2 g every six hours) or the traditional combination of flucloxacillin and benzylpenicillin will cover both of these organisms. The exception to this is in necrotising fasciitis (usually caused by Streptococcus pyogenes or various anaerobes, or both) when the addition of clindamycin is considered mandatory to avoid the risk of apparent resistance attributable to the Eagle effect. $^{52}$ This occurs when Streptococcus pyogenes bacteria enter the stationary phase of bacterial growth and stop expressing the penicillin binding protein (PBP) receptor. This renders penicillin inactive and results in apparent rather than true resistance. Clindamycin overcomes this as it is not dependent on the PBP receptor and acts intracellularly. It is also thought to have an antitoxin effect, which may be clinically important in streptococcal infections. In patients with necrotising fasciitis, Gram negative cover (for example, ciprofloxacin) should also be included in the initial antibiotic regimen until blood and tissue culture results are known.

In patients with risk factors for or who are known to be colonised with MRSA, glycopeptides or other anti-MRSA agents should only be used after reported treatment failure with one of the first line regimens (for example, flucloxacillin) or if the patient is or likely to become severely ill. An important principle in the management of skin and soft tissue infections is to avoid the assumption that bacteria isolated from a skin or wound swab are causal pathogens. For example, Staphylococcus aureus (MSSA or MRSA), Pseudomonas aeruginosa (some of which may be resistant to first line antibiotics), coliforms, and anaerobes are often isolated from skin swabs of chronic venous ulcers of the lower limbs. If the patient does not have surrounding erythema or other evidence of active infection, antibiotics should be avoided. If there is mild cellulitis with no systemic symptoms then a narrow spectrum first line regimen should be prescribed (flucloxacillin). Only in the event of severe sepsis or nonresponding infection, should a broader spectrum antibiotic regimen be used (for example, clindamycin or a glycopeptide, depending on the presence or absence of MRSA, plus ciprofloxacin).

\section{Urinary tract infections}

Most community acquired urinary tract infections (UTIs) are caused by Gram negative coliforms (for example, Escherichia coli, Proteus mirabilis, etc). There have been concerns over recent years about the increasing incidence of resistance against traditionally used antibiotics, such as trimethroprim. Patients with indwelling catheters, those recently exposed to antibiotics, and older patients ( $\geqslant 65$ years) are more likely to have resistant pathogens isolated. ${ }^{53}$ Talan et al showed that a higher proportion of patients clinically and bacteriologically fail treatment in pyelonephritis if they are infected with a resistant organism, even if the length of treatment is doubled (14 days of co-trimoxazole compared with seven days of ciprofloxacin in this study). ${ }^{54}$ It is therefore vital that local epidemiological resistance patterns are accounted for in hospital antibiotic policies. Many hospitals now use alternative antibiotics (for example, co-amoxiclav or cephalosporins or quinolones) for the first line management of patients with UTIs requiring hospital care.

There has also been concern about the over-reliance on urinary dipsticks to confirm the diagnosis rather than the gold standard, which is the presence of significant bacteriuria in a patient with appropriate symptoms, or clinical signs, or both. Given that the presence of asymptomatic bacteriuria is a common finding in the elderly population, to limit 


\section{Key references}

- Barlow G, Nathwani D, Davey P. Appropriate antibiotic prescribing. Proc $R$ Coll Physicians Edinb 2001;31:310-16.

- Communicable Disease Surveillance Centre. Antimicrobial resistance in England and Wales 2000. Public Health Laboratory Service. http://www.hpa.org. uk/infections/topics_az/antimicrobial_resistance/ amr.pdf

- Cosgrove SE, Carmelli Y. The impact of antimicrobial resistance on health and economic outcomes. Clin Infect Dis 2003;36:1433-7.

- Safdar N, Maki DG. The commonality of risk factors for nosocomial colonization and infection with antimicrobial-resistant Staphylococcus aureus, enterococcus, gram-negative bacilli, Clostridium difficile, and Candida. Ann Intern Med 2002;136:834-44.

- Scottish Infection Standards and Strategies Group. Good practice guidance for antibiotic prescribing in hospital. J R Coll Physicians Edinb 2003;33:281-4.

unnecessary antibiotic use, particularly in acutely confused elderly patients who are not otherwise severely unwell, infection at other sites should be excluded and significant bacteriuria confirmed before prescribing antibiotics. ${ }^{55}$ In patients with severe sepsis, a specimen of urine (by catheter if required) should be collected before starting empiric antibiotics. The antibiotic sensitivities of isolates should guide continuing treatment. In women, simple UTIs require only three days of treatment while in men and patients with complicated UTIs or pyelonephritis a minimum of seven days of effective treatment is required (patients with prostatitis require at least four weeks of treatment). ${ }^{454}$

\section{Gram positive bacteraemia}

In the UK, apart from coagulase negative staphylococci, which are usually contaminants secondary to poor blood culture technique, the most commonly isolated Gram positive bacteria from blood cultures are Staphylococcus aureus, Streptococcus pneumoniae, and Lancefield group A, B, and G streptococci (see table 1). ${ }^{11}$ The microbiologist will sometimes be able to inform the clinician which of these is most probable depending on microscopic appearance and initial microbiological tests. The clinician will sometimes be able to determine the most probable source of infection and combined with the early microbiological information this should guide empiric antibiotic treatment. For example, a patient admitted to a medical admissions ward with a history of recent sinusitis and fever and whose blood culture is positive for Gram positive diplococci probably has a Streptococcus pneumoniae bacteraemia. As there is comparatively little penicillin resistance $(7 \%$ in the year 2000$)$ to this organism in the UK and given the evidence discussed above, ${ }^{11}{ }^{24-26}$ this patient can be started with high dose narrow spectrum antipneumococcal treatment (for example, intravenous benzylpenicillin $1.2 \mathrm{~g} / 4 \mathrm{~h}$ or amoxicillin $1 \mathrm{~g} / 6 \mathrm{~h}$ ) pending microbiological sensitivities.

It is also important to consider whether bacteraemia is community or hospital acquired. By convention, bacteraemia occurring after the patient has been in hospital for 48 hours is considered to be hospital acquired. Patients recently discharged from hospital, however, may re-present with a hospital acquired bacteraemia. It is important to establish this, as infections caused by hospital acquired bacteria are much more likely to be resistant to first line agents. For example, in many UK hospitals about one half of all hospital acquired Staphylococcus aureus bacteraemia are methicillin resistant (that is, MRSA; methicillin is a marker of flucloxacillin resistance) whereas most community acquired Staphylococcus aureus bacteraemia in the UK remain methicillin sensitive (that is, MSSA).

As discussed above, Streptococcus pyogenes (Lancefield group A streptococci) is always sensitive to penicillin. There is also no penicillin resistance in group $G$ streptococci and very low resistance $(2 \%)$ in group B streptococci. ${ }^{11}$ There is increasing resistance to erythromycin in all of the above streptococcal species, however, which may be a concern in the future when treating, for example, pneumococcal respiratory tract or streptococcal throat infections in the community with a macrolide antibiotic (erythromycin, clarithromycin, or azithromycin).

\section{Gram negative bacteraemia}

The commonest cause of Gram negative bacteraemia in the UK is Escherichia coli. ${ }^{11}$ In most cases this will be secondary to a UTI, although some patients will have another intraabdominal source of bacteraemia, for example, biliary tract or colonic infection. In the UK, most of these community acquired isolates will be sensitive to the first line antibiotics used for Gram negative bacteria (that is, co-amoxiclav, second/third generation cephalosporins, gentamicin, and ciprofloxacin). ${ }^{11}$ Resistance to these agents in other commonly isolated community acquired Gram negative bacteria,

Table 1 Antimicrobial resistance in commonly isolated pathogens in England and Wales in 2000 (adapted from Communicable Disease Surveillance Centre ${ }^{11}$ )

\begin{tabular}{|c|c|c|c|c|c|c|c|}
\hline \multirow[b]{2}{*}{ Pathogen } & \multicolumn{7}{|c|}{ \% Resistance to various antibiotics } \\
\hline & Pen & Fluclox & Eryth & Cipro & Gent & Ceftaz & Imipen \\
\hline $\begin{array}{l}\text { Gram + bacteraemia } \\
\text { Staphylococcus aureus }\end{array}$ & & 42 & & & & & \\
\hline Streptococcus pneumoniae & 7 & & 15 & & & & \\
\hline Group B streptococcus & 2 & & 5 & & & & \\
\hline Group A streptococcus & 0 & & 4 & & & & \\
\hline Group G streptococcus & 0 & & 12 & & & & \\
\hline Gram-bacteraemia & & & & & & & \\
\hline Escherichia coli & & & & 5 & 3 & $<1$ & \\
\hline Proteus mirabilis & & & & 3 & 1 & $<1$ & \\
\hline Pseudomonas aeruginosa & & & & 15 & 8 & 8 & 5 \\
\hline Klebsiella spp & & & & 10 & 5 & 8 & $<1$ \\
\hline Enterobacter spp & & & & 13 & 6 & 28 & 2 \\
\hline
\end{tabular}

Pen, penicillin; fluclox, flucloxacillin; eryth, erythromycin; cipro, ciprofloxacin; ceftaz, ceftazidime; imipen, imipenem. 
such as Proteus and Klebsiella spp, is also low. Patients who develop bacteraemia in hospital or have recently been discharged from hospital, however, are considerably more likely to be infected with a Gram negative resistant to one or more of the first line agents. ${ }^{28}$ There is also increasing concern about the emergence in UK hospitals of Gram negative bacteria with an extended range of resistance against $\beta$ lactam antibiotics, including $\beta$-lactam/ $\beta$-lactamase inhibitor combinations and third generation cephalosporins (the extended spectrum $\beta$-lactamases or ESBLs). Similar to MRSA, there have been reports of spread outside of hospitals and the subsequent admission of patients with community acquired ESBL infections ${ }^{56}$; this is currently rare in the UK.

Given the above and depending on the epidemiology of resistance in the hospital, patients with confirmed or suspected community or hospital acquired Gram negative bacteraemia who are not severely unwell may be treated initially with the one of the recognised first line Gram negative agents with adjustment of the regimen according to subsequent antibiotic sensitivity testing. To cover Pseudomonas spp and the possibility of resistance, in patients with severe sepsis, however, especially if hospital acquired, an antibiotic regimen with broader activity is required. This can be achieved by either adding gentamicin to a first line agent or by using broad spectrum monotherapy with pipericillin/tazobactam or a carbapenem (for example, meropenem). For confirmed or suspected ESBL bacteraemia, a carbapenem (not pipericillin/tazobactam or ceftazidime) is the drug of choice.

\section{Meningitis}

The successful implementation of immunisation against meningococcal group $\mathrm{C}$ infection has resulted in fewer patients being admitted to hospital with meningococcal meningitis. Nevertheless, there has been a slow, but steady increase in the MICs of meningococcal isolates to penicillin over recent years (meningococci with a MIC $>0.1 \mathrm{mg} / \mathrm{l}$ have increased from $<1 \%$ in 1984 to $18 \%$ in 2000). ${ }^{11}$ As yet, this has not been shown to be of clinical significance in the UK. Because of the blood-brain barrier, penicillin and other $\beta$ lactams (for example, cephalosporins) are less able to penetrate cerebral spinal fluid compared with other tissues (for example, lung). This means that a comparatively small increase in the MICs of meningococci (and pneumococci) may result in treatment failure in meningitis. ${ }^{57}$ Fortunately, the meningococcus remains exquisitely sensitive to third generation cephalosporins, such as cefotaxime and ceftriaxone. It is these that are recommended for the treatment of bacterial meningitis in adults in the British Infection Society's meningitis management pathway. ${ }^{58}$ In PRP, however, this is not the case; the MIC to cephalosporins may also be increased. Unlike in pneumococcal pneumonia, in which large doses of $\beta$-lactams result in lung tissue concentrations high enough to overcome resistance, in PRP meningitis the lower concentration of antibiotic in the cerebrospinal fluid may not be high enough to eradicate infection. In patients with pneumococcal meningitis that have clear risk factors for PRP or confirmed infection therefore, vancomycin should be added to first line therapy. Many infection experts would also add rifampicin.

\section{Malaria}

The epidemiology of resistance to antimalarial drugs is complex and beyond the scope of this review. In the UK, about 2000 cases of imported malaria are diagnosed annually.$^{59}$ An increasing proportion of these cases are caused by Plasmodium falciparum, the life threatening form of the disease. Traditionally, falciparum malaria in the UK has been treated with quinine and Fansidar. In recent years, however, widespread Fansidar resistance has emerged and forthcoming guidance will suggest the combined use of quinine and doxycycline (monotherapy with Malarone or Riamet is an alternative in uncomplicated falciparum infection).$^{60}$ The treatment of all cases of malaria, however, should be discussed with either a regional infectious diseases unit or one of the schools of tropical medicine.

\section{Hospital acquired infections}

Acute physicians and doctors on-call for general medicine are often asked to review patients who may have hospital acquired infection. After admission to hospital, patients are rapidly colonised with bacteria from the hospital's ecology, which are more resistant than their normal flora. ${ }^{61}$ Other bacteria (for example, MRSA) may be transmitted subsequently from other patients via healthcare staff. Although these patients have a higher risk of being infected with an antibiotic resistant pathogen, ${ }^{28}$ this does not mean that broad spectrum agents should automatically be used. Indeed, to do so would further fuel the development of resistance.

The first step is to establish if infection is probable, as there are many potential causes of deterioration in an already hospitalised patient. Postoperative patients, for example, can suffer pulmonary emboli, myocardial infarction, blood loss, and atrial fibrillaton, all of which can cause a systemic inflammatory response and mimic sepsis. It is therefore necessary to take a thorough history (if possible) and perform a careful examination. Common sources of hospital acquired infection include: the skin at the site of an invasive device (for example, peripheral or central intravenous catheters, gastrostomy tubes, etc); the lower respiratory tract (particularly in patients who have difficulties in swallowing or those with nasogastric feeding tubes); the urinary tract; and Clostridium difficile diarrhoea. Bacteraemia is common in patients with invasive devices, especially central intravenous catheters. Deep seated infection secondary to bacteraemia (for example, endocarditis and bone/joint infection) should be searched for on examination. Initial investigations should include peripheral and central intravenous catheter (if applicable) blood cultures, urine culture, and a chest radiograph. Skin sites exuding pus should be swabbed and an MRSA screen performed. Other investigations (for example, an echocardiogram to look for endocarditis) will depend on clinical findings. If the patient has diarrhoea, it is mandatory to send a stool sample for Clostridium difficile toxin, as this is the commonest cause of hospital acquired diarrhoea.

After initial assessment, if infection is clinically unlikely then antibiotics should not be prescribed and microbiological results awaited. If infection is the probable source of deterioration, the severity of illness and the epidemiology of resistance in the hospital should dictate initial antibiotic therapy. In most UK hospitals, for patients that do not have bacteraemia, severe sepsis, or septic shock, this will be flucloxacillin for suspected Gram positive infections (for example, minor skin sepsis) and co-amoxiclav, a second/third generation cephalosporin or ciprofloxacin for Gram negative infections (for example, hospital acquired lower respiratory tract and UTI). For patients with bacteraemia, severe sepsis, or septic shock, a glycopeptide is necessary for Gram positive infections, to cover the possibility of MRSA, and intravenous gentamicin should be added to intravenous co-amoxiclav, a second/third generation cephalosporin, or ciprofloxacin for Gram negative infection. Metronidazole should also be added if anaerobic infection is a concern. Monotherapy with piperacillin/tazobactam or meropenem is an alternative if gentamicin is clinically inappropriate. The recent emergence of Gram negative bacteria producing ESBL enzymes is currently receiving great attention in the infection literature. $^{62}$ Although ESBLs are undoubtedly an emerging threat for UK hospitals, given the low overall incidence, you would 


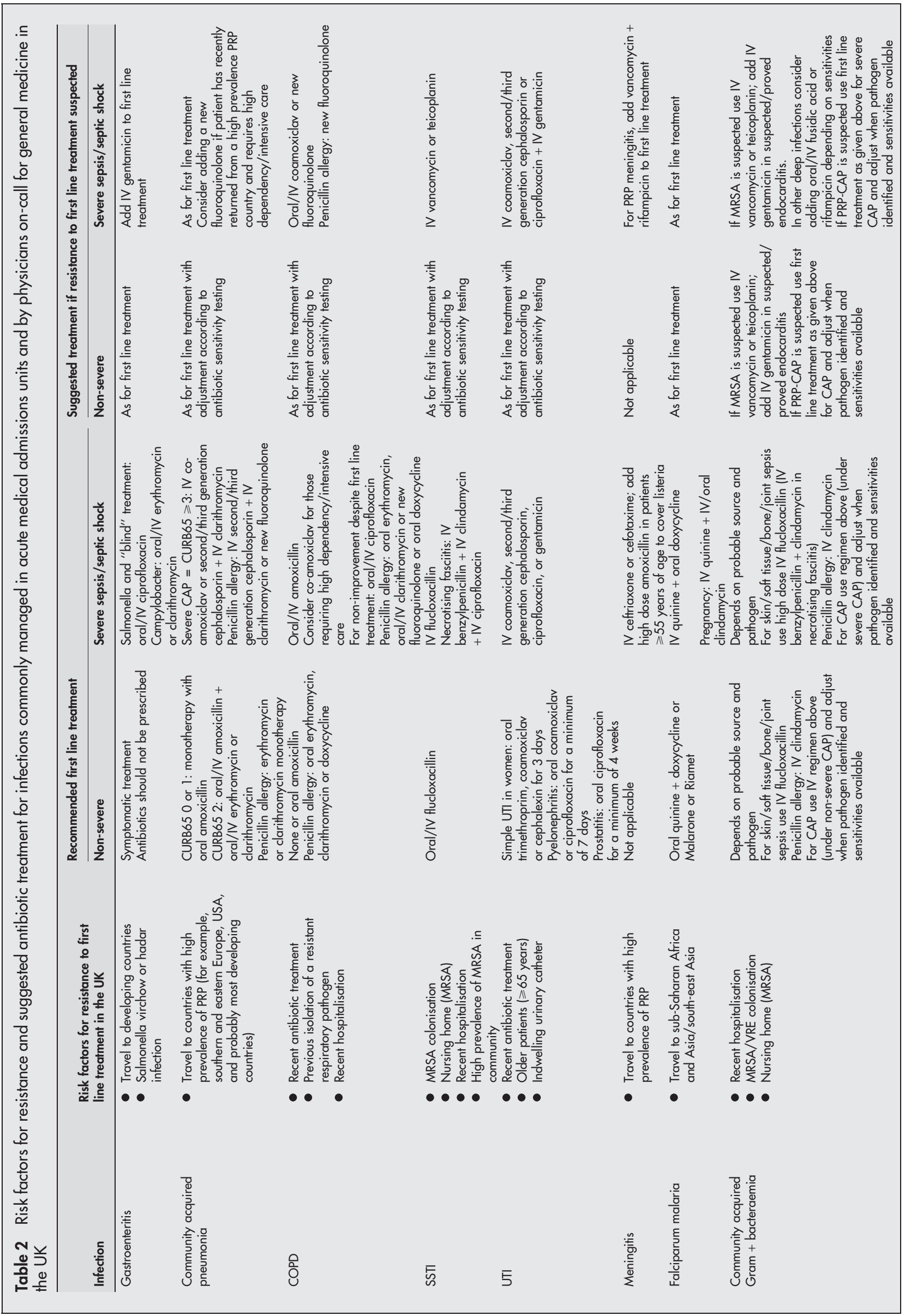




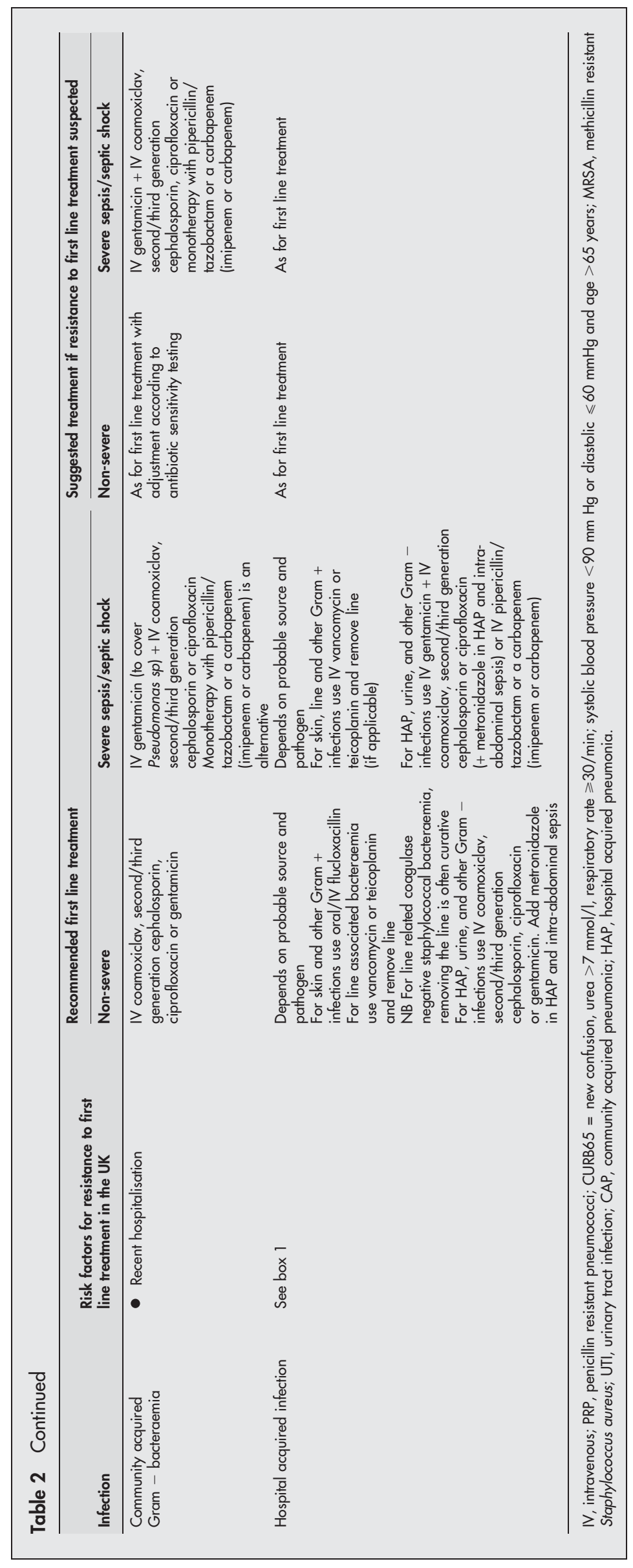




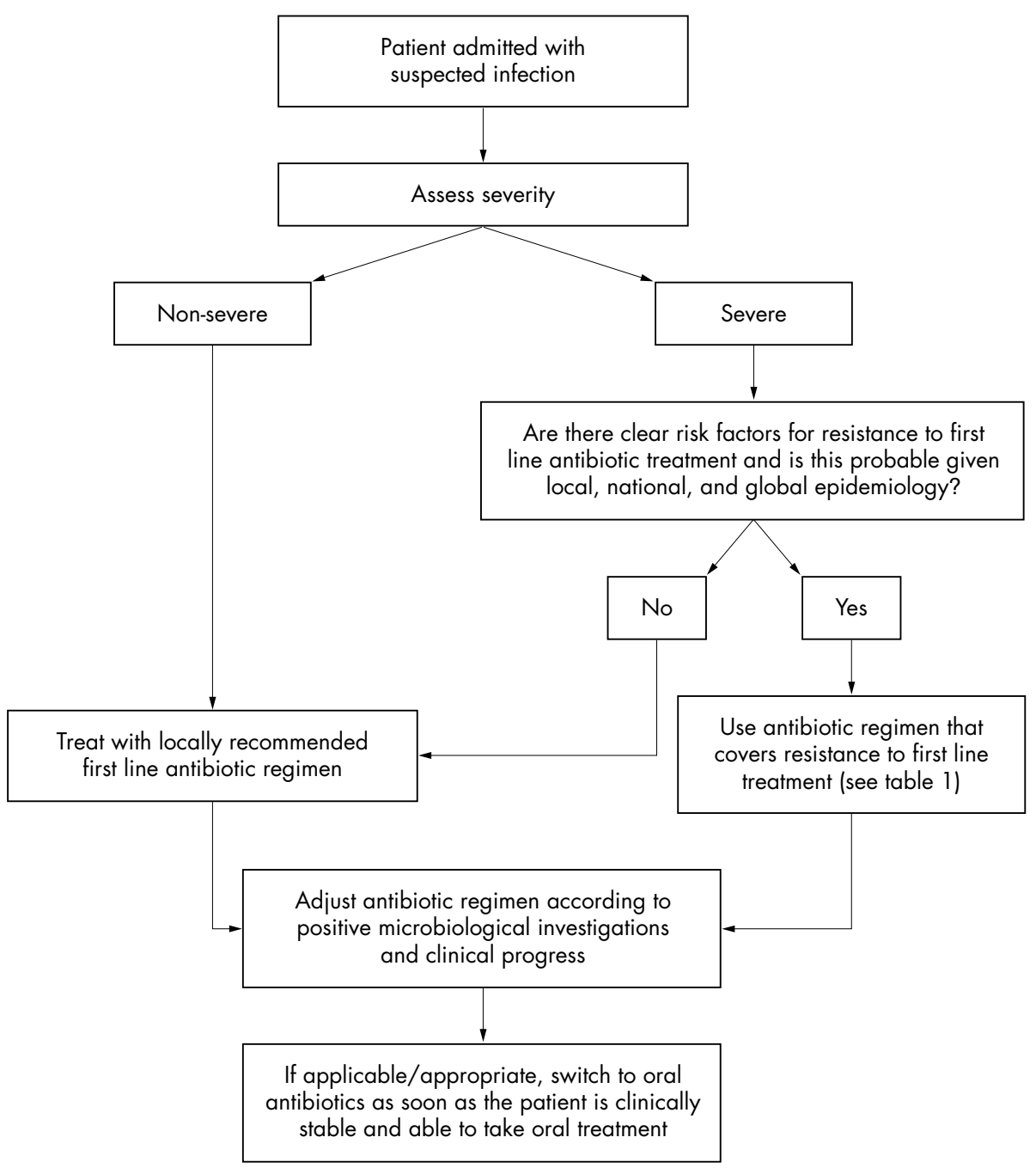

Figure 1 Suggested management pathway for aiding antibiotic selection when resistance may be present.

not currently cover for their possibility in the empiric antibiotic regimen, except where local epidemiological data show that this is necessary.

Table 1 shows antibiotic resistance patterns for common causes of bacteraemia in the UK. Table 2 suggests alternatives to recommended first line antimicrobials when resistance is suspected. Figure 1 shows a management pathway for aiding antibiotic prescribing when resistance is a concern.

\section{CONCLUSIONS}

The use of antibiotics is intimately linked with the development of acquired antibiotic resistance. Prudent antimicrobial prescribing can reduce the development and consequences of resistance. With this in mind, the Scottish Infection Standards and Strategies Group have recently developed minimum standards of care for hospitals in Scotland regarding antibiotic policies and related procedures. ${ }^{63}$ In UK patients hospitalised with community acquired infections, antibiotic resistance should not currently affect empiric prescribing unless the patient has severe sepsis and a specific risk factor(s) for a resistant pathogen. In contrast, resistance is of greater concern in hospital acquired infections. The management of these should be based on initial severity assessment, the probability of a resistant pathogen based on risk factor assessment, and knowledge of local antibiotic resistance patterns.
MULTIPLE CHOICE QUESTIONS (TRUE (T)/FALSE (F); ANSWERS AT END OF REFERENCES)

1. The following statements about the evolution and spread of antibiotic resistance are true:

(A) After admission to hospital, a patient's skin is rapidly colonised with bacteria from the local environment

(B) The spread of resistant microbes in hospitals is mainly attributable to poor hand hygiene

(C) Patients who have taken antibiotics recently are less likely to harbour resistant bacteria

(D) When using $\beta$-lactam antibiotics, an inadequate dosing frequency encourages the evolution of resistance

(E) When using quinolone antibiotics, the use of high doses encourages the evolution of resistance

2. When risk assessing patients for antimicrobial resistance, the following are true:

(A) Compared with a patient who has never been abroad, a patient who has recently returned from Spain with pneumonia is more likely to be infected with a penicillin resistant pneumococcus

(B) Older patients are no more likely to be colonised with a resistant pathogen than younger ones 
(C) MRSA colonisation is more likely in patients with open skin wounds

(D) Most patients colonised with MRSA will develop an MRSA infection in the 18 months after identification

(E) Febrile patients who are known to be colonised with MRSA should be treated with a glycopeptide antibiotic

3. The following are true when interpreting microbiological investigations:

(A) Most patients with a positive stool culture for a Salmonella spp should be treated with oral ciprofloxacin

(B) An otherwise well, confused patient with a positive urine dipstick test for leucocytes and nitrites should be treated with antibiotics

(C) Streptococcus pyogenes is always sensitive to penicillin

(D) Colonisation of chronic leg ulcers with Pseudomonas spp is common and does not necessarily suggest infection

(E) In most hospitals in the UK, about one half of all hospital acquired Staphylococcus aureus infections are resistant to flucloxacillin

4. When managing patients on the acute medical admissions ward, the following statements are true:

(A) Over-treatment of mild infection is unlikely to have important consequences

(B) Under-treatment of severe sepsis rarely leads to poorer outcomes

(C) Empiric antibiotic prescribing should primarily be based on severity assessment and knowledge of local microbial epidemiology

(D) Patients with penicillin resistant pneumococcal pneumonia are unlikely to respond to $\beta$-lactam agents

(E) In pyelonephritis, a seven day course of an antibiotic the pathogen is sensitive to is less effective than a 14 day course of an antibiotic the pathogen is resistant to

5. The following antimicrobial regimens are appropriate for the given clinical scenario

(A) Chloroquine and primaquine for a patient with falciparum malaria

(B) Intravenous coamoxiclav and gentamicin for a patient with severe sepsis and hospital acquired Gram negative bacteraemia

(C) Amoxicillin and clarithromycin for a patient who has recently returned from North America and has nonsevere community acquired pneumonia

(D) A carbapenem (meropenem or imipenem) for a patient with an ESBL producing Gram negative bacteraemia

(E) Amoxicillin for a patient with non-severe pneumonia and a heavy growth of a Klebsiella spp in a sputum sample

\author{
Authors' affiliations \\ G Barlow, Department of Infection and Tropical Medicine, Hull and East \\ Yorkshire Hospitals NHS Trust, Hull, UK \\ D Nathwani, Infection Unit, Tayside University Hospitals NHS Trust, \\ Dundee, UK \\ Funding: none. \\ Competing interests: none declared.
}

\section{REFERENCES}

1 Abboud FM, Wiasbren BA. Correlation between in vitro studies and response to antibiotic therapy in staphylococcal bacteraemia. Arch Intern Med $1959 ; 104: 226-33$.
2 Barlow G, Nathwani D, Davey P. Appropriate antibiotic prescribing. Proc R Coll Physicians Edinb 2001;31:310-16.

3 World Health Organisation. Overcoming antimicrobial resistance. World health report on infectious diseases 2000. http://www. who.int/infectiousdisease-report/2000/index-rpt2000_text.html (accessed 14 Aug 2000).

4 Standing Medical Advisory Committee Sub-Group on Antimicrobial Resistance. The path of least resistance. London: Department of Health, 1998. 5 Wenzel RP. The antibiotic pipeline-challenges, costs and values. NEJM 2004:351:523-24.

6 Kollef MH, Sherman G, Ward S, et al. Inadequate antimicrobial treatment of infections: a risk factor for hospital mortality among critically ill patients. Chest 1999;115:462-74.

7 Ibrahim EH, Sherman G, Ward S, et al. The influence of inadequate antimicrobial treatment of bloodstream infections on patient outcomes in the ICU setting. Chest 2000;118:146-55.

8 Luna CM, Vujacich P, Niederman MS, et al. Impact of BAL data on the therapy and outcome of ventilator-associated pneumonia. Chest 1997; 11 1:676-85.

9 Singh N, Rogers $\mathrm{P}$, Atwood CW, et al. Short-course empiric antibiotic therapy for patients with pulmonary infiltrates in the intensive care unit: a proposed solution for indiscriminate antibiotic prescription. Am J Respir Crit Care Med 2000;162:505-11.

10 Barlow G, Sachdev N, Nathwani D. The use of adult isolation facilities in a UK infectious diseases unit. J Hosp Infect 2002;50:127-32.

11 Communicable Disease Surveillance Centre. Antimicrobial resistance in England and Wales 2000. Public Health Laboratory Service. http:// www.hpa.org.uk/infections/topics_az/antimicrobial_resistance/amr.pdf (accessed 5 Jan 2005).

12 Edlund C, Stark C, Nord CE. The relationship between an increase in $\beta$ lactamase activity after oral administration of three new cephalosporins and protection against intestinal ecological disturbances. J Antimicrob Chemother 1994;34:127-38.

13 Steinke DT, Seaton RA, Phillips G, et al. Prior trimethroprim use and trimethroprim-resistant urinary tract infection: a nested case-control study with multivariate analysis for other risk factors. J Antimicrob Chemother $2001 ; 47: 781-7$

14 Seaton RA, Steinke DT, Phillips G, et al. Community antibiotic therapy, hospitalization and subsequent respiratory tract isolation of Haemophilus influenzae resistant to amoxycillin: a nested case-control study. J Antimicrob Chemother 2000;46:307-9.

15 Magee JT, Pritchard EL, Fitzgerald KA, et al. Antibiotic prescribing and antibiotic resistance in community practice: restrospective study, 1996-8. BMJ 1990;319:1239-40.

16 Seppala H, Klaukk T, Vuopio-Varkila J, et al. The effect of changes in the consumption of macrolide antibiotics on erythromycin resistance in group $A$ streptococci in Finland. Finnish Study Group for Antimicrobial Resistance. NEJM 1997;337:441-6.

17 Murray BE. Vancomycin-resistant enterococcal infections. NEJM 2000;342:710-21.

18 European Antimicrobial Resistance Surveillance System Management Team. European Antimicrobial Resistance Surveillance System Report 2003. http:// www.earss.rivm.nl (accessed 8 Mar 2005).

19 Cosgrove SE, Carmelli Y. The impact of antimicrobial resistance on health and economic outcomes. Clin Infect Dis 2003;36:1433-7.

20 Travers K, Barza M. Morbidity of infections caused by antimicrobial resistant bacteria. Clin Infect Dis 2002;34:S131-4.

21 Cosgrove SE, Sakoulas G, Perencevich EN, et al. A comparison of mortality associated with methicillin-resistant and methicillin-susceptible Staphylococcus aureus bacteremia: a meta-analysis. Clin Infect Dis 2003;36:53-9.

$22 \operatorname{Kim} \mathrm{T}, \mathrm{Oh} \mathrm{PI}, \mathrm{Simor}$ AE. The economic impact of methicillin-resistant Staphylococcus aureus in Canadian hospitals. Infect Control Hosp Epidemiol 2001;22:99-104.

23 Chaix C, Durand-Zaleski I, Alberti C, et al. Control of endemic methicillinresistant Staphylococcus aureus: a cost-benefit analysis in an intensive care unit. JAMA 1999;282:1745-51.

24 Pallares $\mathbf{R}$, Linares J, Vadillo $M$, et al. Resistance to penicillin and cephalosporin and mortality from severe pneumococcal pneumonia in Barcelona, Spain. NEJM 1995;333:474-80.

25 Ewig S, Ruiz M, Torres A, et al. Pneumonia acquired in the community through drug-resistant Streptococcus pneumoniae. Am J Respir Crit Care Med 1999; 159:1835-42.

26 Aspa J, Rajas O, Rodríguez de Castro F, on behalf of the Pneumococcal Pneumonia in Spain Study Group, et al. Drug-resistant pneumococcal pneumonia: clinical relevance and related factors. Clin Infect Dis 2004;38:787-98.

27 Singer ME, Harding I, Jacobs MR, et al. Impact of antimicrobial resistance on health outcomes in the outpatient treatment of adult community-acquired pneumonia: a probability model. J Antimicrob Chemother 2003;51:1269-82.

28 Safdar N, Maki DG. The commonality of risk factors for nosocomial colonization and infection with antimicrobial-resistant Staphylococcus aureus, enterococcus, gram-negative bacilli, Clostridium difficile, and Candida. Ann Intern Med 2002;136:834-44

29 Said-Salim B, Mathema B, Kreiswirth BN. Community-acquired methicillinresistant Staphylococcus aureus: an emerging pathogen. Infect Control Hosp Epidemiol 2003;24:451-5

30 Eady EA, Cove JH. Staphylococcal resistance revisited: community-acquired methicillin resistant Staphylococcus aureus - an emerging problem for the management of skin and soft tissue infections. Curr Opin Infect Dis 2003;16:103-24.

31 Huang SS, Platt R. Risk of methicillin-resistant Staphylococcus aureus infection after previous infection or colonization. Clin Infect Dis 2003;36:281-5. 
32 Solomkin JS, Biornson HS, Cainzos $M$, et al. A consensus statement on empiric therapy for suspected gram-positive infections in surgical patients. Am J Surg 2004;187:134-45.

33 Lim WS, van der Eerden MM, Laing R, et al. Defining community-acquired pneumonia severity on presentation to hospital: an international derivation and validation study. Thorax 2003;58:377-82.

34 Nyström P-O. The systemic inflammatory response syndrome: definitions and aetiology. J Antimicrob Chemother 1998;41(suppl A): 1-7.

35 Barlow G, Nathwani D. Sequential antibiotic therapy. Currr Opin Infect Dis 2000;13:599-607.

36 Drusano GL. Pharmacodynamics of anti-infectives: target delineation and target attainment. In: Finch RG, Greenwood D, Norrby SR, et al, eds. Antibiotic and chemotherapy: anti-infective agents and their use in therapy 8th ed. London: Churchill Livingstone, 2003.

37 BTS guidelines for the management of community-acquired pneumonia in adults. Thorax 2001;56(suppl 4):1-64.

38 Guillemont D, Carbon C, Balkau B, et al. Low dosage and long treatment duration of beta-lactam: risk factors for carriage of penicillin resistant Streptococcus pneumoniae. JAMA 1998;279:365-70.

39 Servais $\mathbf{H}$, Tulkens PM. Stability and compatibility of ceftazidime administered by continuous infusion to intensive care patients. Antimicrob Agents Chemother 2001;45:2643-7.

40 Pfeiffer RR. Structural features of vancomycin. Rev Infect Dis $1981 ; 3$ (suppl):S205-9.

41 Cruciani M, Gatti G, Lazzarini L, et al. Penetration of vancomycin into human lung tissue. J Antimicrob Chemother 1996;38:865-9.

42 Berendt T, Byren I. Bone and joint infection. Clin Med 2004;4:510-18.

43 Farthing $M$, Feldman $R$, Finch $R$, et al. The management of infective gastroenteritis in adults: A consensus statement by an expert panel convened by the British Society for the Study of Infection. J Infect 1996;33:143-52.

44 Lamb VA, Mayhall CG, Spadora AC, et al. Outbreak of Salmonella typhimurium gastroenteritis due to an imported strain resistant to ampicillin, chloramphenicol, and trimethroprim-sulfamethoxazole in a nursery. J Clin Microbiol 1984;20:1076-9.

45 Threlfall EJ, Fisher IS, Berghold C, et al. Trends in antimicrobial drug resistance in Salmonella enterica serotypes typhi and paratyphi A isolated in Europe, 1999-2001. Int J Antimicrob Agents 2003;22:487-91.

46 American Thoracic Society. Guidelines for the management of adults with community-acquired pneumonia. Am J Respir Crit Care Med 2001; 163:1730-54

47 Mandell LA, Marrie TJ, Grossman RF, and the Canadian CommunityAcquired Pneumonia Working Group, et al. Canadian guidelines for the initial management of community-acquired pneumonia: an evidence-based update by the Canadian Infectious Diseases Society and the Canadian Thoracic Society. The Canadian Community-Acquired Pneumonia Working Group. Clin Infect Dis 2000;31:383-421.

48 Bartlett JG, Dowell SF, Mandell LA, et al. Practice guidelines for the management of community-acquired pneumonia in adults. Clin Infect Dis 2000;31:347-82

49 Saint S, Bent S, Vittinghoff E, et al. Antibiotics in chronic obstructive pulmonary-disease exacerbations - a meta-analysis. JAMA 1995;273:957-60.
50 The National Collaborating Centre for Chronic Conditions. Chronic obstructive pulmonary disease: national clinical guideline on management of chronic obstructive pulmonary disease in adults in primary and secondary care. http://wwwthorax.bmijournals.com/content/vol59/suppl_1/ (accessed 4 Mar 2005).

51 Di Perri G, Bonora S. Which agents should we use for the treatment of multidrug-resistant Mycobacterium tuberculosis? J Antimicrob Chemother 2004;54:593-602

52 Eagle $\mathrm{H}$. Experimental approach to the problem of treatment failure with penicillin: group A Streptococcus infection in mice. Am J Med 1952;13:389-99.

53 Wright SW, Wrenn KD, Haynes $M$, et al. Prevalence and risk factors for multidrug resistant uropathogens in ED patients. Am J Emerg Med 2000;18:143-6.

54 Talan DA, Stamm WE, Hooton TM, et al. Comparison of ciprofloxacin (7 days) and trimethroprim-sulfamethoxazole ( 14 days) for acute uncomplicated pyelonephritis in women: a randomized trial. JAMA 2000;283: 1583-9.

55 Nicolle L, on behalf of the SHEA Long Term Care Committee. Urinary tract infections in long-term-care facilities. Infect Control Hosp Epidemiol 2001;22:167-75.

56 Rodriguez-Bano J, Navarro MD, Romero L, et al. Epidemiology and clinical features of infections caused by extended-spectrum beta-lactamase-producing Escherichia coli in non-hospitalized patients. J Clin Microbiol 2004;42:1089-94.

57 John CC. Treatment failure with use of a third-generation cephalosporin for penicillin-resistant pneumococcal meningitis: case report and review. Clin Infect Dis 1994;18:188-93.

58 Begg N, Cartwright KAV, Cohen J, et al. Consensus statement on diagnosis, investigation, treatment and prevention of acute bacterial meningitis in immunocompetent adults. J Infect 1999;39:1-15

59 Travel Health Surveillance Section. Illness associated with foreign travel in England, Wales, and Northern Ireland: a baseline report to 2002. London: Health Protection Agency, http://www.hpa.org.uk/infections/topics_az/ travel/pdf/full_version.pdf (accessed 8 Mar 2005).

60 Wongsrichanalai C, Pickard AL, Wernsdorfer WH, et al. Epidemiology of drug-resistant malaria. Lancet Infect Dis 2002;2:209-18.

61 Powell M, Sanderson PJ. Resistant coagulase-negative staphylococci in hospital patients. J Hosp Infect 1987;9:48-53.

62 Dancer S. Extended spectrum beta-lactamases-are we prepared for the threat? Supplement to Scottish Centre for Infection and Environmental Health Weekly Report, Dec, 2004

63 Scottish Infection Standards and Strategies Group. Good practice guidance for antibiotic prescribing in hospital. J R Coll Physicians Edinb 2003;33:281-4.

\section{ANSWERS}

1. (A) T, (B) T, (C) F, (D) T, (E) F; 2. (A) T, (B) F, (C) T, (D)

F, (E) F; 3. (A) F, (B) F, (C) T, (D) T, (E) T; 4. (A) F, (B) F,

(C) $\mathrm{T}$, (D) $\mathrm{F},(\mathrm{E}) \mathrm{F}$; 5. (A) F, (B) T, (C) T, (D) T, (E) F. 